PROCEEDINGS OF THE

AMERICAN MATHEMATICAL SOCIETY

Volume 135, Number 8, August 2007, Pages 2619-2622

S 0002-9939(07)08760-6

Article electronically published on February 28, 2007

\title{
COFINALITY CHANGES REQUIRED FOR A LARGE SET OF UNAPPROACHABLE ORDINALS BELOW $\aleph_{\omega+1}$
}

\author{
M. C. STANLEY
}

(Communicated by Julia Knight)

\begin{abstract}
In $V$, assume that $\aleph_{\omega}$ is a strong limit cardinal and $2^{\aleph_{\omega}}=\aleph_{\omega+1}$. Let $A$ be the set of approachable ordinals less than $\aleph_{\omega+1}$. An open question of M. Foreman is whether $A$ can be non-stationary in some $\aleph_{\omega}$ and $\aleph_{\omega+1}$ preserving extension of $V$. It is shown here that if $W$ is such an outer model, then $\left\{k<\omega: \mathrm{cf}^{W}\left(\aleph_{k}^{V}\right)=\aleph_{n}^{W}\right\}$ is infinite, for each positive integer $n$.
\end{abstract}

Let $\lambda$ be a infinite cardinal and assume that $2^{\lambda}=\lambda^{+}$. Let $\left\langle a_{\delta}: \delta<\lambda^{+}\right\rangle$list all of the bounded subsets of $\lambda^{+}$. An ordinal $\alpha<\lambda^{+}$is approachable if there exists an unbounded $x \subseteq \alpha$ of order type $\operatorname{cf}(\alpha)$ such that every proper initial segment of $x$ is included in the list $\left\langle a_{\delta}: \delta<\alpha\right\rangle$.

Modulo the non-stationary ideal on $\lambda^{+}$, the definition of "approachable" is independent of the enumeration $\left\langle a_{\delta}: \delta<\lambda^{+}\right\rangle$of bounded subsets of $\lambda^{+}$.

The set of approachable ordinals cannot be very small. An easy observation is that, modulo the non-stationary ideal, every cofinality- $\omega$ ordinal below $\lambda^{+}$is approachable. A result of Shelah [S1] is that if $\lambda$ is singular, then for each regular $\kappa<\lambda$, below $\lambda^{+}$there exists a stationary set of cofinality- $\kappa$ approachable ordinals. The weak square principle $\square_{\lambda}^{*}$ implies that every ordinal below $\lambda^{+}$(modulo the non-stationary ideal) is approachable. Consequently, the statement

$$
\left\{\alpha<\aleph_{\omega+1}: \alpha \text { is unapproachable }\right\} \text { is stationary in } \aleph_{\omega+1},
$$

is a strong "anti- $\square$ " statement.

This paper concerns the special case of $\lambda=\aleph_{\omega}$ under the assumptions that $\aleph_{\omega}$ is a strong limit and that $2^{\aleph_{\omega}}=\aleph_{\omega+1}$. Let us make these assumptions for the remainder of the paper.

Cummings-Foreman-Magidor [CFM2], building on work of Shelah, show that $(\dagger)$ is consistent relative to the existence of a supercompact cardinal. ForemanMagidor [FM] show that the strong transfer principle $\left(\aleph_{\omega+1}, \aleph_{\omega}\right) \rightarrow\left(\aleph_{1}, \aleph_{0}\right)$ implies $(\dagger)$, and Magidor has shown that Martin's Maximum implies $(\dagger)$.

In the case of each of these results, it is the set of cofinality- $\omega_{1}$ unapproachable ordinals that is shown to be stationary. M. Foreman has asked whether the unapproachable ordinals in higher cofinalities might be stationary or, stronger yet,

Received by the editors December 6, 2005 and, in revised form, April 19, 2006 and April 28, 2006 .

2000 Mathematics Subject Classification. Primary 03E05.

Key words and phrases. Approachable ordinal, $I[\lambda]$, cofinality, Erdős-Rado.

Research supported by NSF grant DMS-0501114.

(C)2007 American Mathematical Society 
under suitable large cardinal hypotheses, is it possible that there exists an $\aleph_{\omega}$ and $\aleph_{\omega+1}$-preserving forcing extension of $V$ in which there is a closed unbounded (club) subset of $\aleph_{\omega+1}$ consisting of ordinals that are unapproachable in $V$ ?

This paper shows that if $W$ is an $\aleph_{\omega}$ and $\aleph_{\omega+1}$ preserving extension of $V$ in which there exists a club set of $V$-unapproachable ordinals, then for each positive natural number $n,\left\{k<\omega: \mathrm{cf}^{W}\left(\aleph_{k}^{V}\right)=\aleph_{n}^{W}\right\}$ is infinite.

The following notation will be handy. If $X$ is a set of ordinals, let

$$
\lim (X)=\{\alpha \in X: \alpha=\sup (X \cap \alpha)\}
$$

and, if $\mu$ is regular, let

$$
X^{(\mu)}=\{\alpha \in \lim (X): \operatorname{cf}(\alpha)=\mu\} .
$$

The first lemma is just an observation about a standard proof of the Erdős-Rado Theorem.

Lemma 1. Suppose that $\lambda$ is an infinite cardinal and that $S^{\left(\lambda^{+}\right)} \subseteq \alpha$ is stationary in $\alpha$, where $c f(\alpha)>\mu^{\lambda}$, for cardinals $\mu<c f(\alpha)$. If $F:[S]^{2} \rightarrow \lambda$, then there exists an $F$-homogeneous $H$ such that ot $(H)=\lambda^{+}$and $\sup (H) \in S$.

Proof. Let $\mathfrak{A}=\langle\alpha ; F,<, \delta\rangle_{\delta<\lambda}$. If " $\mathfrak{B}$ ", perhaps decorated, names a substructure of $\mathfrak{A}$, let " $B$ " with the same decoration indicate $\mathfrak{B}$ 's universe.

Say that $\mathfrak{B} \prec \mathfrak{A}$ is $\lambda^{+}$-saturated relative to $\mathfrak{A}$ if $(\mathfrak{B}, b)_{b \in X}$ realizes every type that is realized in $(\mathfrak{A}, b)_{b \in X}$, for all $X \subseteq B$ such that $|X|<\lambda^{+}$. Note that, given an infinite $X \subseteq \alpha$, the least $\mathfrak{B} \prec \mathfrak{A}$ such that $X \subseteq B$ and $\mathfrak{B}$ is $\lambda^{+}$-saturated relative to $\mathfrak{A}$ has cardinality at most $|X|^{\lambda}$.

Let $\left\langle\alpha_{i}: i<\operatorname{cf}(\alpha)\right\rangle$ be increasing and cofinal in $\alpha$. Define $\left\langle\mathfrak{B}_{i}: i<\operatorname{cf}(\alpha)\right\rangle$ by

$$
\mathfrak{B}_{i}=\left\{\begin{array}{c}
\text { the least } \mathfrak{B} \prec \mathfrak{A} \text { such that } \lambda \cup\left\{\alpha_{j}: j<i\right\} \cup \bigcup_{j<i} B_{j} \subseteq B \text { and } \\
\mathfrak{B} \text { is } \lambda^{+} \text {-saturated relative to } \mathfrak{A} \text {. }
\end{array}\right.
$$

Note $\left|B_{i}\right|<\operatorname{cf}(\alpha)$, for $i<\operatorname{cf}(\alpha)$, hence $\sup \left(B_{i}\right)<\alpha$. Also $\sup _{i<\mathrm{cf}(\alpha)}\left(\sup \left(B_{i}\right)\right)=\alpha$. Also, $B_{i}=\bigcup_{j<i} B_{j}$ if $\operatorname{cf}(i) \geq \lambda^{+}$. Thus $\left\{\sup \left(B_{i}\right): i<\operatorname{cf}(\alpha)\right\}$ is $\lambda^{+}$-closed and unbounded in $\alpha$.

Fix a limit ordinal $i<\operatorname{cf}(\alpha)$ of cofinality $\lambda^{+}$such that $\sup \left(B_{i}\right) \in S$. Set $\eta=$ $\sup \left(B_{i}\right)$. Choose $\beta_{\xi}$ by recursion on $\xi<\lambda^{+}$to be such that $\beta_{\xi} \in B_{i}$ and $\beta_{\xi}$ realizes in $\left(\mathfrak{B}_{i}, \beta_{\zeta}\right)_{\zeta<\xi}$ the same type that $\eta$ does in $\left(\mathfrak{A}, \beta_{\zeta}\right)_{\zeta<\xi}$, and $\left\langle\beta_{\xi}: \xi<\lambda^{+}\right\rangle$ is cofinal in $\eta$. Then $P=\left\{\beta_{\xi}: \xi<\lambda^{+}\right\}$is cofinal in $\eta$ and is $F$-prehomogeneous: If $\beta<\beta^{\prime}<\beta^{\prime \prime}$ lie in $P$, then $F\left(\beta, \beta^{\prime}\right)=F\left(\beta, \beta^{\prime \prime}\right)$.

Finally, choose $H \subseteq P$ cofinal in $\eta$ and such that, for some $\delta<\lambda$ we have $f(\beta)=\delta$, for all $\beta \in H$, where $f(\beta)=F\left(\beta, \beta^{\prime}\right)$, for all $\beta^{\prime}>\beta$ in $P$.

If $\lambda<\mu<\kappa$ are infinite cardinals, with $\lambda$ and $\kappa$ both regular, say that $S \subseteq \kappa$ $\lambda$-reflects at cofinality $>\mu$ if

$$
\left\{\alpha<\kappa: \operatorname{cf}(\alpha)>\mu \text { and } S^{(\lambda)} \cap \alpha \text { is stationary in } \alpha\right\}
$$

is stationary in $\kappa$.

Proposition 2. Assume that $\aleph_{\omega}$ is a strong limit cardinal. For $n<\omega$, the set of unapproachable ordinals less than $\aleph_{\omega+1}$ does not $\aleph_{n+1}$-reflect at cofinality $>2^{\aleph_{n}}$. 
Proof. Fix an enumeration $\vec{x}=\left\langle x_{\alpha}: \alpha<\aleph_{\omega+1}\right\rangle$ of the bounded subsets of $\aleph_{\omega+1}$. For $\alpha<\aleph_{\omega+1}$, let $f_{\alpha}: \alpha \rightarrow \aleph_{\omega}$ be one-to-one, and set $\vec{f}=\left\langle f_{\alpha}: \alpha<\aleph_{\omega+1}\right\rangle$. Let $S$ be the set of unapproachable (with respect to $\vec{x}$ ) ordinals less than $\aleph_{\omega+1}$. Fix Skolem functions and set

$$
M_{i}=\text { the least } M \prec\left\langle H_{\aleph_{\omega+1}} ; \vec{f}, \vec{x}\right\rangle \text { such that } \aleph_{\omega} \cup\left\{M_{j}: j<i\right\} \subseteq M,
$$

for $i<\aleph_{\omega+1}$.

We maintain that $\left\{M_{i} \cap \aleph_{\omega+1}: i<\aleph_{\omega+1}\right\}$ witnesses that $S$ does not $\aleph_{n+1^{-}}$ reflect at cofinality $>2^{\aleph_{n}}$, for all $n$. Fix $n<\omega$. For the sake of a contradiction, suppose that there exists a limit ordinal $i$ such that $\operatorname{cf}(i)>2^{\aleph_{n}}$ and $S^{\left(\aleph_{n+1}\right)} \cap \alpha$ is stationary in $\alpha$, where $\alpha=M_{i} \cap \aleph_{\omega+1}$. Let $F:[S \cap \alpha]^{2} \rightarrow \omega$ by

$$
F(\gamma, \beta)=\text { the least } k \text { such that } f_{\beta}(\gamma)<\omega_{k} .
$$

Note that if $\aleph_{m}<\operatorname{cf}(\alpha)$, then $\aleph_{m}^{\aleph_{n}} \leq \aleph_{m} \cdot 2^{\aleph_{n}}<\operatorname{cf}(\alpha)$. By the previous lemma, let $H \subseteq S \cap\left\{M_{j} \cap \aleph_{\omega+1}: j \in \lim (i)\right\}$, be $F$-homogeneous, say to color $k$, and such that ot $(H)=\aleph_{n+1}$ and $\eta=\sup (H) \in S$. We shall reach a contradiction by seeing that $\eta$ is approachable with respect to $\vec{x}$.

Let $j \in \lim (i)$ be such that $\eta=M_{j} \cap \aleph_{\omega+1}$. It suffices to see that $H \cap \delta \in M_{j}$, for $\delta<\eta$, since $\left\langle x_{\gamma}: \gamma<\eta\right\rangle$ enumerates all bounded subsets of $\eta$ that lie in $M_{j}$. Say $\beta \in H \backslash \delta$. Then $f_{\beta}^{\prime \prime}(H \cap \delta) \subseteq \omega_{k}$. Hence $f_{\beta}^{\prime \prime}(H \cap \delta) \in M_{j}$. But then $H \cap \delta \in M_{j}$, since $\beta \in M_{j}$.

Suppose that $W$ is an $\aleph_{\omega}$ and $\aleph_{\omega+1}$ preserving outer model of $V$. Our goal is to see that if in $W$ there is a club set of $V$-unapproachable ordinals below $\aleph_{\omega+1}$, then $\left\{k<\omega: \mathrm{cf}^{W}\left(\aleph_{k}^{V}\right)=\aleph_{n}^{W}\right\}$ is infinite, for $n>0$. In Corollaries 3 and 4 this implication is interpolated by the following combinatorial principle:

There exists a sequence $\left\langle C_{\alpha}: \alpha \in C\right\rangle$ with the following three properties:

(1) $C$ is club in $\aleph_{\omega+1}$.

(*) (2) $C_{\alpha}$ is club in $\alpha$, if $\operatorname{cf}(\alpha)>\omega$.

(3) If $\operatorname{cf}^{V}(\alpha)>\left(2^{\aleph_{n}}\right)^{V}$, then every ordinal $\delta \in C_{\alpha}$ such that $\operatorname{cf}^{V}(\delta) \neq \omega$ satisfies $\operatorname{cf}^{V}(\delta)>\aleph_{n+1}^{V}$.

Remark. The proof of Corollary 3 below is easily modified to yield $(*)$ meeting two additional requirements

(4) ot $\left(C_{\alpha}\right)=\mathrm{cf}^{W}(\alpha)$ and

(5) $C_{\alpha} \subseteq C \cap \alpha$.

These are not needed for the promised conclusion of this paper in Corollary 4 .

Corollary 3. Suppose that $\aleph_{\omega}$ is a strong limit cardinal in $V$ and that $W$ is an $\aleph_{\omega}$ and $\aleph_{\omega+1}$ preserving outer model of $V$ in which

$$
U=\left\{\alpha<\aleph_{\omega+1}: V \models " \alpha \text { is unapproachable" }\right\}
$$

has a club subset. Then $W$ satisfies $(*)$.

Proof. Let $E \subseteq U$ be club in $\aleph_{\omega+1}$. By the previous proposition, we may assume that if $\alpha \in E$ and $\mathrm{cf}^{V}(\alpha)>\left(2^{\aleph_{n}}\right)^{V}$, then $\left\{\delta \in \lim (U \cap \alpha): \mathrm{cf}^{V}(\delta)=\aleph_{n+1}^{V}\right\}$ is nonstationary in $\alpha$. For $\alpha \in \lim (E)$, let $E_{\alpha}$ be club in $\alpha$ and such that if $\left(2^{\aleph_{n}}\right)^{V}<$ $\operatorname{cf}^{V}(\alpha)$ and $\delta \in E_{\alpha}$ and $\operatorname{cf}^{V}(\delta)=\aleph_{n+1}$, for some $n$, then $\delta \notin U$. Set $C=\lim (E)$ and $C_{\alpha}=E \cap E_{\alpha}$, for $\alpha \in C$. 
Corollary 4. Assume that $V$ is an inner model of $W$ with the same $\aleph_{\omega}$ and $\aleph_{\omega+1}$, and that $W$ satisfies $(*)$. Then

$$
\left\{k<\omega: c f^{W}\left(\aleph_{k}^{V}\right)=\aleph_{n}^{W}\right\}
$$

is infinite, for all $n>0$.

Proof. Suppose not. Suppose that $n, k<\omega$ and that if $\mathrm{cf}^{W}\left(\aleph_{i}^{V}\right)=\aleph_{n}^{W}$, then $i \leq k$. Choose $\alpha \in C$ such that $\mathrm{cf}^{W}(\alpha)>\left(2^{\aleph_{k}}\right)^{V}, \aleph_{n}^{W}$. Let $\delta$ be the $\omega_{n}^{\text {th }}$ element of $C_{\alpha}$. Then $\mathrm{cf}^{W}(\delta)=\aleph_{n}^{W}$, but $\mathrm{cf}^{V}(\delta)>\aleph_{k+1}^{V}$.

\section{REFERENCES}

[HJS] A. Hajnal, I. Juhász, and S. Shelah, Splitting strongly almost disjoint families, Trans. Amer. Math. Soc. 295, no. 1 (1986), 360-387. MR0831204 (87i:03098)

[S1] S. Shelah, On successors of singular cardinals, Logic Colloquium '78 (M. Boffa et al., eds.), Stud. Log. Found. Math. 97, North Holland Publ. Co., Amsterdam-New York, 1979, pp. 357-380. MR0567680(82d:03079)

[S2] _ Cardinal Arithmetic, Oxford Logic Guides 29, Oxford, $1994 . \quad$ MR.1318912 (96e:03001)

[C] J. Cummings, Collapsing successors of singulars, Proc. Amer. Math. Soc. 125, 27032709. MR 1416080 (97j:03091)

[FM] M. Foreman and M. Magidor, A very weak square principle, Jour. Sym. Log. 62, no. 1 (1997), 175-196. MR1450520 (98i:03062)

[CFM1] J. Cummings, M. Foreman, and M. Magidor, Canonical structure in the universe of set theory: Part I, Ann. Pur. Appl. Log. 129, nos. 1-3 (2004), 211-243. MR2078366 (2005k:03105)

[CFM2] - Canonical structure in the universe of set theory: Part II (to appear).

Mathematics Department, San Jose State University, San Jose, California 95192

E-mail address: stanley@math.sjsu.edu 